\title{
Novel modelling of ultra-compact X-ray binary evolution - stable mass transfer from white dwarfs to neutron stars
}

\author{
Rahul Sengar ${ }^{1 \star}$, Thomas M. Tauris ${ }^{2,1}$, Norbert Langer ${ }^{1}$, Alina G. Istrate ${ }^{3}$ \\ 1 Argelander-Institut für Astronomie, Universität Bonn, Auf dem Hügel 71, D-53121 Bonn, Germany \\ 2 Max-Planck-Institut für Radioastronomie, Auf dem Hügel 69, D-53121 Bonn, Germany \\ 3 Center for Gravitation, Cosmology, and Astrophysics, Dept. of Physics, Uni. of Wisconsin-Milwaukee, Milwaukee, WI, 53201, USA
}

Accepted 2017 April 26. Received 2017 April 26; in original form 2017 March 7

\begin{abstract}
Tight binaries of helium white dwarfs (He WDs) orbiting millisecond pulsars (MSPs) will eventually "merge" due to gravitational damping of the orbit. The outcome has been predicted to be the production of long-lived ultra-compact X-ray binaries (UCXBs), in which the WD transfers material to the accreting neutron star (NS). Here we present complete numerical computations, for the first time, of such stable mass transfer from a He WD to a NS. We have calculated a number of complete binary stellar evolution tracks, starting from pre-LMXB systems, and evolved these to detached MSP+WD systems and further on to UCXBs. The minimum orbital period is found to be as short as 5.6 minutes. We followed the subsequent widening of the systems until the donor stars become planets with a mass of $\sim 0.005 M_{\odot}$ after roughly a Hubble time. Our models are able to explain the properties of observed UCXBs with high helium abundances and we can identify these sources on the ascending or descending branch in a diagram displaying mass-transfer rate vs. orbital period.
\end{abstract}

Key words: binaries: close — X-rays: binaries — stars: mass-loss — stars: neutron - white dwarfs - pulsars: general

\section{INTRODUCTION}

The detection of radio millisecond pulsars (MSPs) in close orbits with helium white dwarfs (He WDs) raises interesting questions about their future destiny. One example is PSR J0348+0432 (Antoniadis et al. 2013) which has an orbital period of $2.46 \mathrm{hr}$. Due to continuous emission of gravitational waves, this system will "merge" in about 400 Myr. However, rather than resulting in a catastrophic event, once the WD fills its Roche lobe, the outcome is expected to be a long-lived ultracompact X-ray binary (UCXB, Webbink 1979; Nelson et al. 1986; Podsiadlowski et al. 2002; Nelemans et al. 2010; van Haaften et al. 2012a; Heinke et al. 2013). These sources are tight low-mass X-ray binaries (LMXBs) observed with an accreting neutron star (NS) and a typical orbital period of less than 60 min. Because of the compactness of UCXBs, the donor stars are constrained to be either a WD, a semidegenerate dwarf or a helium star (Rappaport et al. 1982).

Depending on the mass-transfer rate, the UCXBs are classified in two categories: persistent and transient sources. Until now, only 14 UCXBs have been confirmed (9 persistent, 5 transient), and an additional 14 candidates are

^ E-mail: rsengar@astro.uni-bonn.de known. Therefore, we can infer that UCXBs are difficult to detect or represent a rare population. Earlier studies (e.g. Istrate et al. 2014b) have suggested the need for extreme fine tuning of initial parameters (stellar mass and orbital period of the LMXB progenitor systems) in order to produce an UCXB from an LMXB system. Analytical investigations by van Haaften et al. (2012a) and van Haaften et al. (2012b) on the evolution of UCXBs with NS or black hole accretors reveal that these systems can evolve to orbital period of 100-110 min, thereby explaining the existence of the so-called diamond planet pulsar (Bailes et al. 2011).

UCXBs are detected with different chemical compositions in the spectra of their accretion discs (e.g. H, He, C, $\mathrm{O}$ and Ne, Nelemans et al. 2010). To explain this diversity requires donor stars which have evolved to different levels of nuclear burning and interior degeneracy, and therefore to different scenarios for the formation of UCXBs. Since a large fraction of the UCXBs are found in globular clusters, some of these UCXB systems could also have formed via stellar exchange interactions (Fabian et al. 1975). For a $1.4 M_{\odot}$ NS

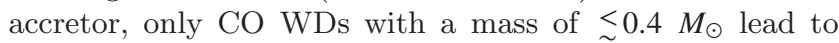
stable UCXB configurations (van Haaften et al. 2012a), although recent hydrodynamical simulations suggest that this critical WD mass limit could be lower (Bobrick et al. 2017).

Here we focus on numerical computations covering, for 
the first time, complete evolution of NS-main sequence star binaries which evolve into LMXBs and later produce UCXBs with a He WD donor star. The evolution is terminated when the donor reaches a mass of $\sim 0.005 M_{\odot}$ (about 5 Jupiter masses) after several Gyr, with a radius close to the maximum radius of a cold planet.

In Section 2, we present the applied stellar evolution code, as well as key assumptions on binary interactions and a summary of our applied model. The results of our calculations are presented in Section 3. A comparison with the observed UCXBs systems is give in Section 4, and finally we further discuss and summarise our results in Section 5 .

\section{NUMERICAL CODE AND INITIAL SETUP}

We applied the MESA code (Modules for Experiments in Stellar Astrophysics, Paxton et al. 2015, and references therein) for calculating the evolution of NS-main sequence star binaries. Our initial binary system consists of a $1.3 M_{\odot}$ NS (treated as a point mass) and a zero-age main-sequence (ZAMS) donor star of mass $M_{2}=1.4 M_{\odot}$ with solar chemical composition $(X=0.70, Y=0.28$ and $Z=$ 0.02 ). We investigated a range of initial orbital periods of $P_{\text {orb, }} \simeq 2-5$ days, with a total of 40 models. These orbits were assumed to be circular and synchronized. We assumed standard loss of orbital angular momentum due to magnetic braking (only significant during the LMXB phase), gravitational wave (GW) radiation and mass loss (e.g. Tauris \& van den Heuvel 2006). We modelled the latter via Roche-lobe overflow (RLO) according to the isotropic re-emission model (van den Heuvel 1994; Tauris \& Savonije 1999), in which matter flows from the donor star to the accreting NS in a conservative manner and a fraction (here $\beta=0.70$, see discussions in Istrate et al. 2014b) is ejected from the vicinity of the NS, and at all times ensuring sub-Eddington mass-accretion rates $\left(\left|\dot{M}_{2}\right|<\dot{M}_{\text {Edd }} \simeq 3.0 \times\right.$ $\left.10^{-8} M_{\odot} \mathrm{yr}^{-1}\right)$. For the initial phase of the mass transfer in the UCXB stage (once the He WD remnant initiates RLO to the NS), the mass-transfer rate is super-Eddington, and thus the NS accretion rate is limited to $\dot{M}_{\text {Edd }}$. For simplicity, and to avoid too many free parameters, we do not include the possibility of a circumbinary (CB) disc (Shao \& Li 2012), nor do we consider irradiation of the donor star via pulsar winds or photons.

\section{RESULTS}

\section{$3.1 \quad \mathrm{LMXB} /$ pre-UCXB evolution}

Fig. 1 shows the orbital period evolution as a function of age for several LMXBs with a range of $P_{\text {orb,i }}=2.2-5.0$ days and magnetic braking index $\gamma=5$ (Istrate et al. 2014b). For these close-orbit binaries, magnetic braking efficiently shrinks the orbits such that the companion star is forced to initiate RLO within $1-3$ Gyr. Based on the classification of Pylyser \& Savonije (1988) and Istrate et al. (2014b), we divide the orbital evolution of the resulting LMXBs into diverging, intermediate and converging systems. Notably, we find a narrow range of $P_{\text {orb, } i}$ for which LMXB systems detach and produce a He WD in a tight orbit (marked by

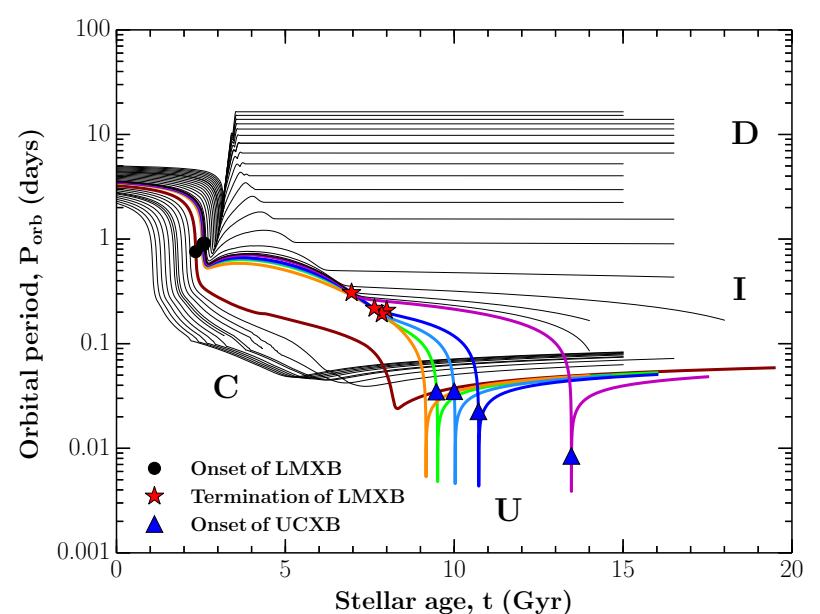

Figure 1. Orbital period evolution of LMXBs. The outcome is either diverging (D) or intermediate (I) systems, converging (C) systems, or binaries (U) which detach, produce He WDs and evolve into UCXBs. See also fig. 2 in Istrate et al. (2014b). The colour codings of the tracks are identical to those in Figs. 2-4.

U, see also Istrate et al. 2014b, for discussions). Such systems are observable as radio MSP binaries with typical $P_{\text {orb }} \simeq 2-9 \mathrm{hr}$ (e.g. PSRs J0751+1807 and J0348+0432, Ergma et al. 1997; Antoniadis et al. 2013). These MSP binaries will shrink their orbits further by GW radiation; most of them to the extent that their He WDs are forced to fill their Roche lobe within a total age of less than a Hubble time. At this stage the systems become UCXBs, typically when $P_{\text {orb }} \simeq 10-50 \mathrm{~min}$, cf. Fig. 2 . As a result of the $\left(M_{\mathrm{WD}}, P_{\mathrm{orb}}\right)-$ relation for He WDs (Tauris \& Savonije 1999; Istrate et al. $2014 \mathrm{~b}$ ) all our UCXBs initially have $M_{\mathrm{WD}} \simeq 0.15-0.17 M_{\odot}$.

\subsection{UCXB evolutionary tracks}

In Fig. 2, we plot evolutionary tracks for the UCXB phase (i.e. post-LMXBs) of six systems with values of $P_{\text {orb,i }}$ between 3.25 and 3.52 days, cf. coloured tracks in Fig. 1. Several features of UCXB formation can be seen from Fig. 2 which we now discuss in more detail.

Firstly, it is evident that LMXBs with $P_{\text {orb,i }}$ below a certain threshold value (depending on initial values of $M_{2}, M_{\mathrm{NS}}$, chemical composition and treatment of magnetic braking; here $~ 3.45$ days) will never detach from RLO to produce a He WD. Their donor stars still possess a significant hydrogen content - even in their cores and due to their very small nuclear burning rates they still have a mixture of hydrogen and helium when they finally become degenerate near the orbital period minimum, $P_{\text {orb }}^{\min } \simeq 10-85 \mathrm{~min}$ (Paczynski \& Sienkiewicz 1981; Rappaport et al. 1982; Nelson et al. 1986; Podsiadlowski et al. 2002; van der Sluys et al. 2005). These converging systems become hydrogen-rich UCXBs and are most likely the progenitor systems of the so-called black widow MSPs (e.g. Ergma et al. 1998; Benvenuto et al. 2012; Chen et al. 2013). For our two hydrogen-rich systems with $P_{\text {orb, } \mathrm{i}}=3.25$ days $\left(\right.$ red track) and $P_{\text {orb, }}=3.44$ days (orange track), we find $P_{\text {orb }}^{\min } \simeq 35 \mathrm{~min}$ and $8 \mathrm{~min}$, respectively.

Secondly, for UCXBs with He WD donors, the larger the value of $P_{\text {orb, } \mathrm{i}}$, the smaller is $P_{\text {orb }}$ at the onset of the 


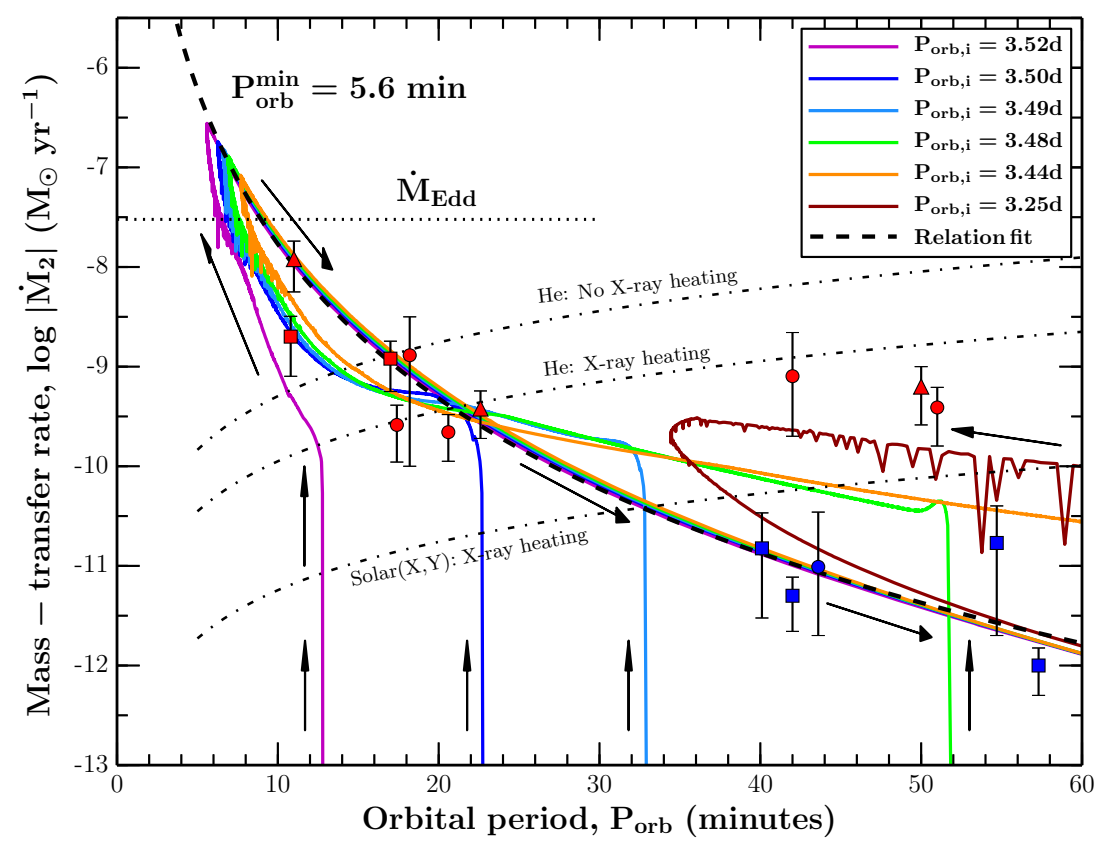

Figure 2. Evolutionary tracks of our modelling of the UCXB phase in the $\left(P_{\text {orb }}, \dot{M}_{2}\right)$-plane, calculated for six different values of $P_{\text {orb, }}$, with RLO from the He WD, following the detached epoch of an MSP-WD system - except for the systems with $P_{\text {orb, }}=3.25$ and 3.44 days which never detach completely in their transition from an LMXB to an UCXB. The UCXB evolution (see black arrows) begins on the vertical tracks, when the He WD initiates RLO, and decreases $P_{\text {orb }}$ due to GWs while climbing up the ascending branch until the tip of the track at $P_{\mathrm{orb}}^{\mathrm{min}}$, before the system settles on the common declining branch (dashed line) while $P_{\text {orb }}$ steadily increases on a Gyr timescale. More details of $\dot{M}_{2}$ and the donor stars are found in Figs. 3 and 4. The observed UCXBs (Heinke et al. 2013) are shown with red (persistent source) and blue (transient source) symbols. UCXBs marked with squares, triangles and circles correspond to systems with no information of spectra, with helium and without helium lines, respectively. The dot-dashed lines indicate accretion disk instability thresholds, depending on X-ray heating and chemical composition. The dotted line is $\dot{M}_{\text {Edd }}$ for a NS accreting helium.

UCXB phase. The reason is that in wider binaries He WDs have larger masses (Tauris \& Savonije 1999); and, more important, since in wider systems it takes longer time for GWs to cause the He WDs to fill their Roche lobe, they will be less bloated (Istrate et al. 2014a), i.e. more compact (and colder) by the time they reach the onset of the UCXB phase.

Thirdly, we identify a unique pattern in the tracks of these UCXBs (see black arrows). They begin on the vertical tracks, when the He WD initiates RLO, and continue decreasing $P_{\text {orb }}$ due to GWs while climbing up the ascending branch until the tip of the track at $P_{\text {orb }}^{\min }$. For our He WD UCXBs we find typically $P_{\text {orb }}^{\min } \simeq 5-7$ min. Following $P_{\text {orb }}^{\min }$, which coincides with a maximum value of $\left|\dot{M}_{2}\right| \simeq 10 \dot{M}_{\text {Edd }}$ (see also Fig. 3), all systems settle on the common declining branch while $P_{\text {orb }}$ steadily increases on a Gyr timescale, with the relation: $\log \left|\dot{M}_{2} / M_{\odot} y r^{-1}\right|=-5.15 \cdot \log \left(P_{\text {orb }} / \min \right)-2.62$.

The shape of the UCXBs tracks can be understood from the ongoing competition between GW radiation and orbital expansion caused by mass transfer/loss. The reason that the maximum value of $\left|\dot{M}_{2}\right|$ coincides with $P_{\text {orb }}^{\text {min }}$ is partly that the He WD donor stars are fully degenerate, which means that their mass-radius exponent is negative (Fig. 4c), whereby they expand in response to mass loss (at least after their residual hydrogen-rich envelope of a few $10^{-3} M_{\odot}$ has been removed, Kaplan et al. 2012). The onset of RLO leads not only to very high mass-transfer rates (Fig.3) but also to an outward acceleration of the orbital size, as a result of the small mass ratio $(q \simeq 0.1)$ between the two stars, such that at some point the rate of orbital expansion dominates over that of shrinking due to GW radiation. As the orbits widen further, the value of $\left|\dot{M}_{2}\right|$ decreases and the strength of GW radiation levels off due to its steep dependence on orbital separation and the systems settle on the common declining branch while the orbit expands at a continuously slower pace. An analogy of our described UCXB models can be made to RLO in double WD systems (Kaplan et al. 2012).

Our final $M-R$ tracks (Fig. 4c) terminating at 5 Jupiter masses $\left(\sim 0.005 M_{\odot}\right)$ are in good agreement with (within $5 \%$ of) the adiabatic helium models of Deloye \& Bildsten (2003) and the cold helium models of Zapolsky \& Salpeter (1969). For a comparison to $M-R$ tracks of cold planets, see Fig. $4 \mathrm{~d}$. The He WD donors never crystallise but remain Coulomb liquids with $\Gamma \leq 35$. In all our models, the final mass of the (post) UCXB NS is $1.70 \pm 0.01 M_{\odot}$, reflecting the assumed NS birth mass (here $1.3 M_{\odot}$ ) and the accretion efficiency.

\section{COMPARISON TO OBSERVATIONS}

At first sight in Fig. 2, we notice that our UCXB tracks can explain the location of the observed systems quite well. The data were taken from Heinke et al. (2013) and the error bars on $\left|\dot{M}_{2}\right|$ reflect bolometric correction uncertainty from the observed X-ray luminosity. The seven persistent 

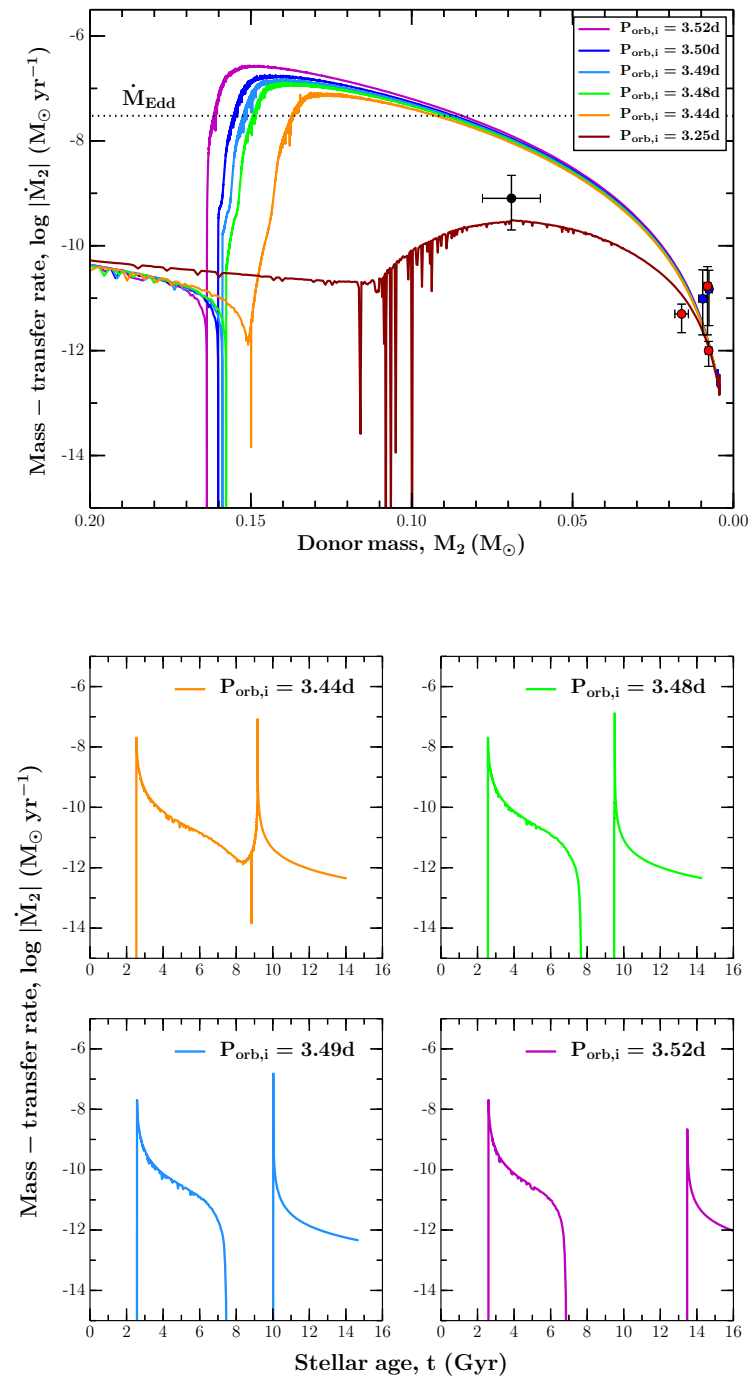

Figure 3. Upper panel: mass-transfer rate, $\left|\dot{M}_{2}\right|$ as a function of donor star mass $\left(M_{2}\right)$ for the UCXB tracks shown in Fig. 2. The observed WD donor masses of six known accreting X-ray MSP UCXBs (five systems are transient, Patruno \& Watts 2012) are marked in red and blue colours which correspond to He and CO WDs. The masses of three WD companions are very similar to one another and cluster at the same point. The WD marked in black colour could either be a WD or a helium star. Lower panels: four of the above tracks plotted in $\left(t,\left|\dot{M}_{2}\right|\right)$-diagrams.

and short-orbital period UCXBs $\left(P_{\text {orb }} \simeq 10-25 \mathrm{~min}\right)$ are either located on the ascending or the descending branch. A simple way to distinguish between the two options is the sign of $\dot{P}_{\text {orb }}$. Unfortunately, the intrinsic value is difficult to derive in practice since many of the UCXBs are located in dense globular clusters, thereby suffering from acceleration in the cluster potential or gravitational perturbations from a nearby object. Moreover, a CB disc may cause $\dot{P}_{\text {orb }}<0$ on the declining branch (Jiang et al. 2017). Statistically, however, UCXBs are much more likely to be on the declining branch since the temporal evolution is a lot slower along this branch (Deloye \& Bildsten 2003; Nelemans et al. 2010).

The three persistent sources with $P_{\text {orb }} \simeq 40-50 \mathrm{~min}$ are best described by an LMXB system which evolves continuously into an UCXB without forming a detached WD (cf. red track for $P_{\text {orb }, \mathrm{i}}=3.25$ days). Alternatively, for a WD donor, a CB disc model can also account for these sources as a result of a significant increase in $\left|\dot{M}_{2}\right|$ (Heinke et al. 2013).

All the five transient systems likely populate the declining branch of the evolutionary tracks. Due to their wider orbits, the radius of their accretion disc is larger and its temperature lower, which causes thermal viscous instabilities and thus a transient behavior (Lasota et al. 2008). Heinke et al. (2013) suggested that this might be the reason why so relatively few UCXBs are seen in wide orbits with $P_{\text {orb }}>30$ min (keeping in mind that UCXBs should accumulate in wide orbits over time). The transient behavior allows radio MSPs to turn on, whereby the "radio ejection mechanism" (Burderi et al. 2002) can prevent further accretion. However, pulsar-wind irradiation of the donor may operate, possibly until $M_{2}<0.004 M_{\odot}$ (if beaming is favourable), at which point the star is likely to undergo tidal disruption (Ruderman \& Shaham 1985) (however, see also Priedhorsky \& Verbunt 1988); potentially leaving behind pulsar planets (Martin et al. 2016). Our evolutionary tracks are terminated just before that when $M_{2} \simeq 0.005 M_{\odot}$ and $P_{\text {orb }}=70-80 \mathrm{~min}$.

To model even wider UCXBs with He WD donors, will probably require irradiation effects to be included. Whereas these effects apparently have little effect on the UCXB evolution in an $\left(M_{2}, P_{\text {orb }}\right)$-diagram (Benvenuto et al. 2012), they do accelerate the evolution of these systems (van Haaften et al. 2012b), which is needed to understand some of the observed relatively wide-orbit (post-UCXB) MSP binaries with very small companion star masses.

So far, we have not discussed the chemical composition of the observed spectra of the UCXB systems which holds the key to understanding their origin (Nelemans et al. 2010). Although the nature of the donor stars has only been established firmly in some cases, it seems already now quite clear that a variety of progenitor models is needed to explain their origin. Our modelling presented here can account for the UCXBs with helium (or hydrogen) lines in their spectra.

\section{SUMMARY}

We have used MESA to calculate the complete evolution of close binary systems leading to the formation and evolution of UCXBs. This includes numerical calculations (to our knowledge, for the first time) of stable mass transfer from a WD to an accreting NS. In this work, we have concentrated on an initial binary with a relatively massive ZAMS donor star of $1.4 M_{\odot}$. This allows for producing UCXBs at $P_{\text {orb }}^{\mathrm{min}}$ within $10 \mathrm{Gyr}$. In another recent work, even more massive donor stars have been suggested to produce UCXBs (Chen \& Podsiadlowski 2016). We also performed additional modelling using $M_{2}=1.2 M_{\odot}$ and $\beta=0.3$. The evolutionary tracks of these systems closely resemble those plotted in Fig. 2, although in this case no UCXBs are produced before a total age of 11.6 Gyr. A particular uncertainty in the first part of our calculations is the modelling of magnetic braking. It is evident that Nature produces tight LMXBs, CVs and NS-WD binaries via this channel, but the calibration of 

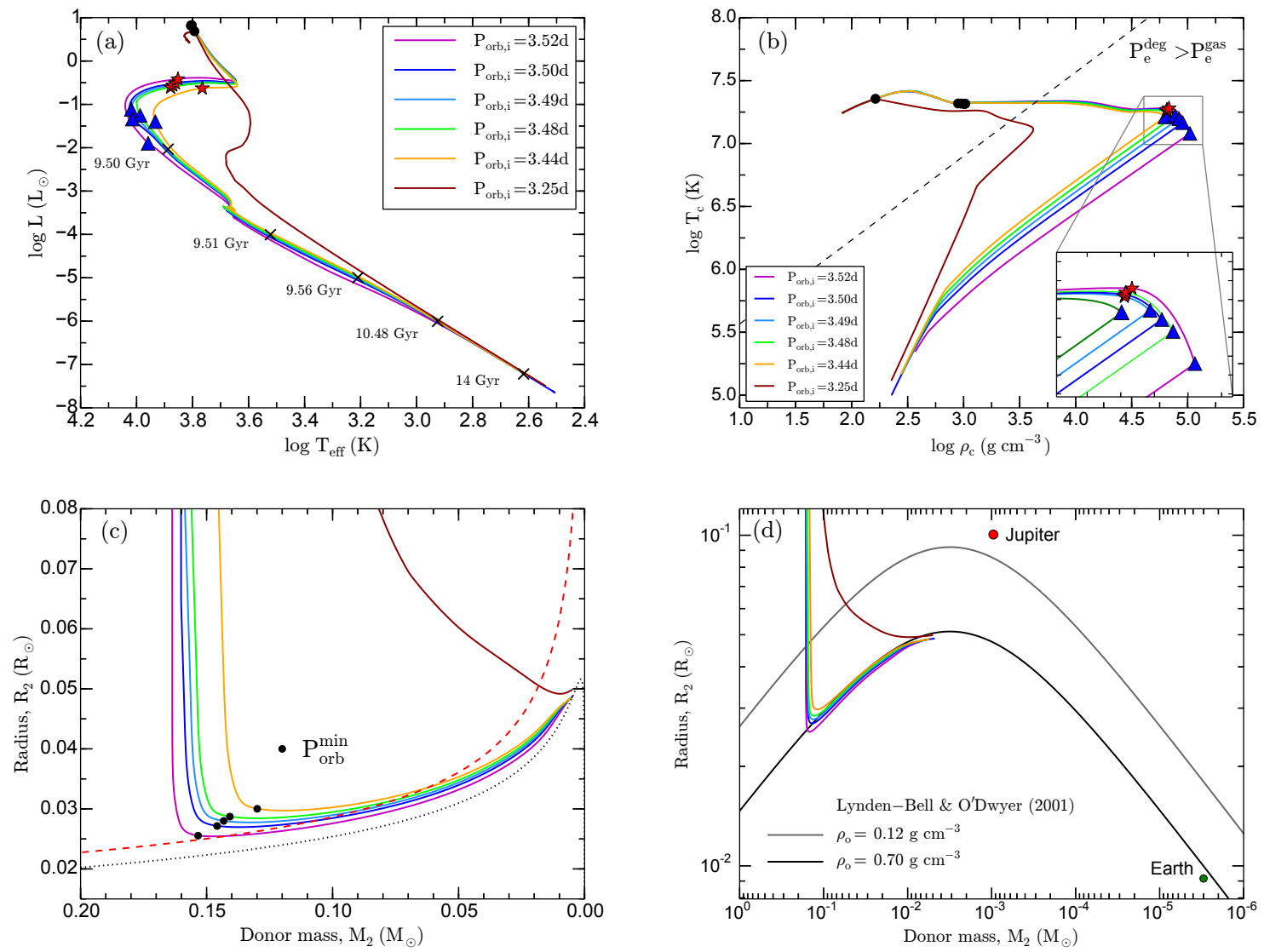

Figure 4. Detailed properties of UCXB donor stars. The four plots displayed are as follows: HR-diagram with stellar ages for $P_{\text {orb }, \mathrm{i}}=$ 3.48 days (upper-left panel, a), central temperature vs. central mass density (upper-right panel, b), stellar radius vs. mass (lower panels, c and d). Common symbols are: black circles (onset LMXB RLO), red stars (termination LMXB RLO) and blue triangles (onset UCXB RLO). The dashed line in panel b separates non-degenerate matter from degenerate matter. The red dashed and black dotted lines in panel c are theoretical mass-radius relations: $R_{\mathrm{WD}}=0.013 R_{\odot} \cdot\left(M_{\mathrm{WD}} / M_{\odot}\right)^{-1 / 3}$ and that of Eggleton used in Rappaport et al. (1987). The two $M-R$ tracks plotted in panel d (grey and black curves) are scaled from eq. (34) in Lynden-Bell \& O'Dwyer (2001) for cold planets.

the effect as well as e.g. the required depth of the convective envelope remains uncertain (Istrate et al. 2014b).

To explain the full population of UCXBs, one needs to perform similar computations leading to donor stars evolved to different degrees of nuclear burning and which therefore have different chemical compositions (i.e. hydrogen-rich dwarf stars, naked helium stars, He WDs or CO WDs). Whether such computations (with or without a commonenvelope phase) are also possible for CO WDs (or hybridCO WDs) remains to be shown.

\section{ACKNOWLEDGEMENTS}

RS thanks AIfA, University of Bonn, for funding during this MSc project and Pablo Marchant for help with MESA. We thank Craig Heinke, John Antoniadis and the referee, Lennart van Haaften, for very useful comments.

\section{REFERENCES}

Antoniadis J., Freire P. C. C., Wex N., Tauris T. M., Lynch R. S., van Kerkwijk M. H., Kramer M., et al. 2013, Science, 340, 448
Bailes M., et al., 2011, Science, 333, 1717

Benvenuto O. G., De Vito M. A., Horvath J. E., 2012, ApJ, 753, L33

Bobrick A., Davies M. B., Church R. P., 2017, MNRAS, 467, 3556

Burderi L., D'Antona F., Burgay M., 2002, ApJ, 574, 325

Chen W.-C., Podsiadlowski P., 2016, ApJ, 830, 131

Chen H.-L., Chen X., Tauris T. M., Han Z., 2013, ApJ, 775, 27

Deloye C. J., Bildsten L., 2003, ApJ, 598, 1217

Ergma E., Lundgren S. C., Cordes J. M., 1997, ApJ, 475, L29

Ergma E., Sarna M. J., Antipova J., 1998, MNRAS, 300, 352

Fabian A. C., Pringle J. E., Rees M. J., 1975, MNRAS, 172, 15p

Heinke C. O., Ivanova N., Engel M. C., Pavlovskii K., Sivakoff G. R., Cartwright T. F., Gladstone J. C., 2013, ApJ, 768, 184

Istrate A. G., Tauris T. M., Langer N., Antoniadis J., 2014a, A\&A, 571, L3

Istrate A. G., Tauris T. M., Langer N., 2014b, A\&A, 571, A45

Jiang L., Chen W.-C., Li X.-d., 2017, ArXiv: 1702.03100,

Kaplan D. L., Bildsten L., Steinfadt J. D. R., 2012, ApJ, 758, 64

Lasota J.-P., Dubus G., Kruk K., 2008, A\&A, 486, 523

Lynden-Bell D., O’Dwyer J. P., 2001, ArXiv: astro-ph/0104450,

Martin R. G., Livio M., Palaniswamy D., 2016, ApJ, 832, 122

Nelemans G., Yungelson L. R., van der Sluys M. V., Tout C. A., 2010, MNRAS, 401, 1347

Nelson L. A., Rappaport S. A., Joss P. C., 1986, ApJ, 304, 231

Paczynski B., Sienkiewicz R., 1981, ApJ, 248, L27

Patruno A., Watts A. L., 2012, preprint, (arXiv:1206.2727)

Paxton B., et al., 2015, ApJS, 220, 15 


\section{L6 Sengar et al.}

Podsiadlowski P., Rappaport S., Pfahl E. D., 2002, ApJ, 565, 1107

Priedhorsky W. C., Verbunt F., 1988, ApJ, 333, 895

Pylyser E., Savonije G. J., 1988, A\&A, 191, 57

Rappaport S., Joss P. C., Webbink R. F., 1982, ApJ, 254, 616

Rappaport S., Ma C. P., Joss P. C., Nelson L. A., 1987, ApJ, 322,842

Ruderman M. A., Shaham J., 1985, ApJ, 289, 244

Shao Y., Li X.-D., 2012, ApJ, 745, 165

Tauris T. M., Savonije G. J., 1999, A\&A, 350, 928

Tauris T. M., van den Heuvel E. P. J., 2006, Formation and evolution of compact stellar X-ray sources. pp 623-665

Webbink R. F., 1979, ApJ, 227, 178

Zapolsky H. S., Salpeter E. E., 1969, ApJ, 158, 809

van Haaften L. M., Nelemans G., Voss R., Wood M. A., Kuijpers J., 2012a, A\&A, 537, A104

van Haaften L. M., Nelemans G., Voss R., Jonker P. G., 2012b, A\&A, 541, A22

van den Heuvel E. P. J., 1994, in Shore S. N., Livio M., van den Heuvel E. P. J., Nussbaumer H., Orr A., eds, Saas-Fee Advanced Course 22: Interacting Binaries. pp 263-474

van der Sluys M. V., Verbunt F., Pols O. R., 2005, A\&A, 431, 647

This paper has been typeset from a $\mathrm{T}_{\mathrm{E}} \mathrm{X} / \mathrm{LATEX}$ file prepared by the author. 